\title{
Tumor-targeting efficacy of a BF211 prodrug through hydrolysis by fibroblast activation protein- $\alpha$
}

\author{
Xiao-ping CHAl ${ }^{1, \#}$, Guang-long SUN ${ }^{2, \#}$, Yan-fen FANG ${ }^{1}$, Li-hong $\mathrm{HU}^{2, *}$, Xuan LIU ${ }^{3, *}$, Xiong-wen ZHANG ${ }^{1, *}$ \\ ${ }^{1}$ Shanghai Engineering Research Center of Molecular Therapeutics and New Drug Development, School of Chemistry and Molecular \\ Engineering, East China Normal University, Shanghai 200241, China; ${ }^{2}$ Jiangsu Key Laboratory for Functional Substance of Chinese \\ Medicine, Jiangsu Collaborative Innovation Center of Chinese Medicinal Resources Industrialization, Stake Key Laboratory Cultivation \\ Base for TCM Quality and Efficacy, School of Pharmacy, Nanjing University of Chinese Medicine, Nanjing 210023, China; ${ }^{3}$ Renji \\ Hospital, School of Medicine, Shanghai Jiaotong University, Shanghai 200127, China
}

\begin{abstract}
BF211, a bufalin (BF) derivative, exhibits stronger anti-cancer activity than BF but with potential cardiotoxicity. Fibroblast activation protein- $\alpha(F A P \alpha)$ is a membrane-bound protease specifically expressed by carcinoma-associated fibroblasts, thus has been used for the selective delivery of anticancer agents. In this study, we used a FAP $\alpha$-based prodrug strategy to synthesize a dipeptide (Z-GlyPro)-conjugated BF211 prodrug named BF211-03. BF211-03 was hydrolyzed by recombinant human FAP $\alpha$ (rhFAP $\alpha$ ) and cleaved by homogenates of human colon cancer HCT-116 or human gastric cancer MGC-803 xenografts. In contrast, BF211-03 showed good stability in plasma and in the homogenates of FAP $\alpha$-negative normal tissues, such as heart and kidney. In HCT-116 and MGC-803 cells with low levels of FAP $\alpha$ expression, BF211-03 displayed a lower in vitro cytotoxicity than BF211 with approximately 30 to 40 -fold larger $\mathrm{IC}_{50}$ values, whereas in human breast cancer MDA-MB-435 cells with high levels of FAP $\alpha$ expression, the IC ${ }_{50}$ value difference between BF211-03 and BF211 was small (approximately 4-fold). Although the cytotoxicity of BF211-03 against tumor cells was dramatically decreased by the chemical decoration, it was restored after cleavage of BF211-03 by rhFAP $\alpha$ or tumor homogenate. In HCT-116 tumorbearing nude mice, doubling the dose of BF211-03, compared with BF211, caused less weight loss, but showing similar inhibitive effects on tumor growth. Our results suggest that BF211-03 is converted to active BF211 in tumor tissues and exhibits anti-tumor activities in tumor-bearing nude mice. FAP $\alpha$-targeted BF211-03 displays tumor selectivity and may be useful as a targeting agent to improve the safety profile of cytotoxic natural products for use in cancer therapy.
\end{abstract}

Keywords: bufalin; BF211; fibroblast activation protein- $\alpha$; prodrug; human cancer cells; xenograft

Acta Pharmacologica Sinica (2018) 39: 415-424; doi: 10.1038/aps.2017.121; published online 9 Nov 2017

\section{Introduction}

Bufalin (BF) is one of the main effective components of the traditional Chinese medicine ChanSu, the dried secretion from the skin and parotid venom glands of Bufo bufo gargarizans Cantor or Bufo melanostictus Schneider. BF211 is a derivative of $\mathrm{BF}$ that exhibits stronger anti-cancer effects than $\mathrm{BF}$ toward various cancer cells and lower toxicity in mice, as shown in previous reports ${ }^{[1,2]}$. The synthesis and anti-cancer use of BF211 had been granted a patent in China (Authorized Announcement No CN 102532235B). However, as a cytotoxic agent, BF211 might induce toxicity in normal tissues, particu-

\footnotetext{
\#These authors contributed equally to this work.

${ }^{*}$ To whom correspondence should be addressed.

E-mail xwzhang@sat.ecnu.edu.cn (Xiong-wen ZHANG); Ihhu@simm.ac.cn (Li-hong HU); liuxuan@renji.com (Xuan LIU)

Received 2017-06-14 Accepted 2017-08-01
}

larly the heart, because this drug is a type of cardiac steroid. The toxicity of BF211 in normal tissues would strongly impede its development as a new anti-cancer drug.

FAPa, a type II transmembrane serine protease, is a tumorassociated antigen that is highly expressed in stromal fibroblasts. Tumor stroma accounts for more than $90 \%$ of the tumor mass in colon, breast and pancreatic carcinomas ${ }^{[3,4]}$. The expression of FAPa is strongly restricted to carcinomaassociated fibroblasts. There is almost no detectable expression of FAPa in normal healthy adult tissues, although FAPpositive cells have been observed during wound healing and embryogenesis, and in areas of chronic inflammation and fibrosis $^{[5]}$. Carcinoma-associated fibroblasts secrete growth factors that promote tumor growth, angiogenesis, and the recruitment of pro-tumorigenic inflammatory cells ${ }^{[6-11]}$. Because of its relative tumor-restricted expression and unique proteolytic activity, FAPa is considered as a tumor-associated 
protease for the selective delivery of anticancer agents, such as doxorubicin ${ }^{[12-15]}$.

In the present study, to improve the tumor targeting of BF211, we designed a BF211 prodrug, named BF211-03, which is cleaved by FAPa into BF211. BF211-03, dipeptide (Z-GlyPro)-conjugated BF211, was synthesized by decorating BF211 with the specific tripeptide $\mathrm{N}, \mathrm{N}$-dimethyl-Gly-Gly-Pro-OH. Here, we report the generation of BF211-03 and characterization of its in vitro and in vivo antitumor activities. The in vitro cytotoxic effects of BF211-03 and BF211 in various human cancer cells and heart-derived rat $\mathrm{H} 9 \mathrm{C} 2$ cells were compared. The in vivo effects of BF211-03 and BF211 on tumor growth and body weight were also observed in nude mice inoculated with human HCT-116 colon cancer cells.

\section{Materials and methods}

\section{Chemicals and materials}

$\mathrm{ChanSu}$, a traditional Chinese medicine comprising the dried secretions from the skin and parotid venom glands of Bufo bufo gargarizans Cantor or Bufo melanostictus Schneider, was obtained from Shanghai Tongrentang Pharmacy (Shanghai, China). As previously described, bufalin (BF) was isolated from ChanSu ${ }^{[16]}$, and BF211 was synthesized ${ }^{[2]}$. Subsequently, BF211-03 was synthesized by decorating BF211 with the specific tripeptide N,N-dimethyl-Gly-Gly-Pro-OH. The chemical structures of BF, BF211 and BF211-03 are shown in Figure 1A. The synthetic route for preparing BF211-03 conjugates is depicted in Figure 1B. L-Proline benzyl ester was used to prepare the specific tripeptide $\mathrm{N}, \mathrm{N}$-dimethyl-Gly-Gly-Pro-OH through a series of synthesis reactions. The conjugate BF21103 has a molecular weight of $751 \mathrm{~g} / \mathrm{mol}$. The purity of BF21103 used in the present study was over $99 \%$. Stock solutions of BF211 and BF211-03 were prepared in DMSO at a concentration of $10 \mathrm{mmol} / \mathrm{L}$ in enzymatic and cell-based assays. For animal studies, BF211 and BF211-03 were dissolved in 5\% Klucel EF $+5 \%$ ethanol $+90 \%$ normal saline, and the stock solution was freshly prepared every 2 days. Recombinant human FAPa (rhFAPa) was purchased from R\&D Systems (Minneapolis, MN, USA).

\section{Cell culture}

The human HCT-116 colon cancer cell line, MGC-803 gastric cancer cell line, MDA-MB-435 breast cancer cell line and rat H9C2 cardiomyocyte cell line were purchased from the Cell Resource Center of Shanghai Institutes for Biological Sciences, Chinese Academy of Sciences (Shanghai, China) and maintained in growth medium at $37^{\circ} \mathrm{C}$ in a $5 \% \mathrm{CO}_{2}$ atmosphere, as recommended. The medium was supplemented with penicillin (100 units $/ \mathrm{mL})$ and streptomycin $(100 \mathrm{mg} / \mathrm{L})$. For experiments, the cells were used during the logarithmic growth phase. All materials used for cell culture were obtained from HyClone (GE Healthcare Life Sciences HyClone Laboratories, South Logan, Utah, USA).

In vitro hydrolysis of BF211-03 by recombinant FAP $\alpha$ protein The hydrolysis of BF211-03 by rhFAPa protein was assessed after incubation of $100 \mu \mathrm{mol} / \mathrm{L}$ BF211-03 with $\mathrm{rhFAPa}$ at $37^{\circ} \mathrm{C}$ in Tris-buffer ( $\mathrm{pH}$ 7.4). First, rhFAPa at different concentrations $(1,2$, and $8 \mu \mathrm{g} / \mathrm{mL})$ was used to identify the minimal effective dose of rhFAPa. Subsequently, the time-dependent cleavage of BF211-03 by rhFAPa was observed by incubation of $100 \mu \mathrm{mol} / \mathrm{L}$ BF211-03 with $2 \mu \mathrm{g} / \mathrm{mL}$ rhFAPa for $1.5,3,6$, 12 or $24 \mathrm{~h}$. Prodrug hydrolysis was monitored by collecting samples at each time point, then analyzing samples by using high-performance liquid chromatography (HPLC; Waters 2695 Alliance LC System). The following HPLC profile was used for the detection of BF211-03 and BF211: UV-detection at 298 $\mathrm{nm}$; Column: KR100-C18 Kromasil column $(5 \mu \mathrm{mol} / \mathrm{L}, 150 \times$ $4.6 \mathrm{~mm}^{2}$ ); flow rate: $1.0 \mathrm{~mL} / \mathrm{min}$; eluent: acetonitrile/ $0.5 \mathrm{vol} \%$ formic acid in water; program: $20 \%-75 \%$ acetonitrile within 20 min (gradient elution); and injection: $10 \mu \mathrm{L}$.

\section{Ex vivo hydrolysis of BF211-03 by using plasma and tissue} homogenates from tumor-bearing nude mice

Blood plasma, tumor tissue and normal tissues, including heart and kidney, were harvested from nude mice bearing HCT-116 or MGC-803 tumors. To prepare the tissue homogenates, $200 \mathrm{mg}$ of tissue was homogenized with $800 \mu \mathrm{L}$ of phosphate-buffered saline (PBS). To assess the hydrolysis of BF211-03 ex vivo, $100 \mu \mathrm{mol} / \mathrm{L}$ BF211-03 was mixed with 400 $\mu \mathrm{L}$ of plasma or tissue homogenates and incubated at $37^{\circ} \mathrm{C}$ in a shaking incubator for $12 \mathrm{~h}$. Subsequently, the samples were collected, processed and measured using HPLC as described above.

\section{Hydrolysis of BF211-03 in cultured cells}

Hydrolysis of BF211-03 was also assessed in cultured HCT116, MGC-803, MDA-MB-435, and H9C2 cells. Briefly, the cells were seeded onto a $6-\mathrm{cm}$ plate at a cell density of $12 \times 10^{5}$ cells per well. After overnight growth, the cell culture medium was changed to fresh medium $(2 \mathrm{~mL})$ containing the prodrug BF211-03 (10 $\mu \mathrm{mol} / \mathrm{L})$. After incubation for $8 \mathrm{~h}$, the culture medium was collected and centrifuged at $3000 \mathrm{r} / \mathrm{min}$ for $5 \mathrm{~min}$ at room temperature. The supernatant was collected and transferred to tubes containing $2 \mathrm{~mL}$ of methylene dichloride. The tubes were vigorously shaken, and subsequently, the organic layer was collected. The extraction was repeated three times. After solvent evaporation and dissolution of the residue in $150 \mu \mathrm{L}$ of methanol, the sample was filtered $(0.22$ $\mu \mathrm{m})$ and analyzed by using HPLC as described above.

\section{Western blot analysis of the FAP $\alpha$ levels}

Western blotting assays were conducted as previously described $^{[1,17,18]}$ to examine the level of FAPa in tissues and cultured cells. The protein samples of tissues or cells were prepared in RIPA buffer containing protease and phosphatase inhibitors (Thermo Fisher Scientific, Waltham, MA, USA), and the protein levels were quantified using a BCA protein assay kit. FAPa was detected using a sheep polyclonal antibody against FAPa (\#AF3715, 1:3000) from R\&D Systems and HRP-linked rabbit anti-goat IgG (\#RAG007, 1:5000) from MultiSciences Biotech Co Ltd (Hangzhou, China). $\beta$-actin 
was detected using an HRP-linked goat polyclonal antibody against $\beta$-actin (\#sc-1615, 1:1000; Santa Cruz, Dallas, TX, USA).

\section{Cytotoxicity assay}

The cytotoxicity of BF211-03 or BF211 in cultured cells was assessed using a CCK8 assay as previously described ${ }^{[19]}$. Briefly, cells in logarithmic growth phase were seeded onto a 96-well tissue culture plate (Thermo Fisher Scientific, Waltham, MA, USA) at a density of 2000, 1500, 4000 or 1000 cells/well for the HCT-116, MGC-803, MDA-MB-435 and $\mathrm{H} 9 \mathrm{C} 2$ cell line, respectively. After cultivation overnight to allow adherence, the cells were treated with 0.1\% DMSO (solvent control), BF211 or BF211-03 at various concentrations for $72 \mathrm{~h}$. After treatment, CCK-8 stock solution diluted in RPMI$1640(100 \mu \mathrm{L})$ was added to each well. The $O D_{450}$ was subsequently measured with SpectraMax M5 (Molecular Devices, $\mathrm{CA}, \mathrm{USA})$. The inhibition rate was calculated as $\left(O D_{\text {control cells }}{ }^{-}\right.$ $\left.O D_{\text {treated cells }}\right) / O D_{\text {control cells }} \times 100 \%$. The $\mathrm{IC}_{50}$ values were calculated through concentration-response curve fitting using the four-parameter method.
To examine whether the hydrolysis of BF211-03 restored the cytotoxicity of BF211, the cytotoxic effects of BF211-03, BF21103 incubated with PBS, BF211-03 incubated with recombinant rhFAPa, BF211-03 incubated with HCT-116 tumor tissue homogenate, BF211, BF211 incubated with PBS, BF211 incubated with recombinant rhFAPa and BF211 incubated with HCT-116 tumor tissue homogenate were compared. Briefly, BF211-03 or BF211 at $100 \mu \mathrm{mol} / \mathrm{L}$ was incubated with rhFAPa $(8 \mu \mathrm{g} / \mathrm{mL}$ rhFAPa for $24 \mathrm{~h})$ or tumor homogenate $(12 \mathrm{~h})$. The hydrolyzed products were collected and filtered $(0.22 \mu \mathrm{m})$. Subsequently, the hydrolyzed products were diluted to different concentrations with cell culture medium. The cytotoxic effects of the samples at gradient dilutions were assessed in cultured HCT-116 cells by using the CCK8 method as described above.

\section{In vivo anti-tumor activity and influence on body weight}

The in vivo anti-tumor activity and influence on body weight of BF211-03 were observed in nude mice with HCT-116 xenografts. Briefly, female nude immunodeficient Balb/c-nu-nu

A

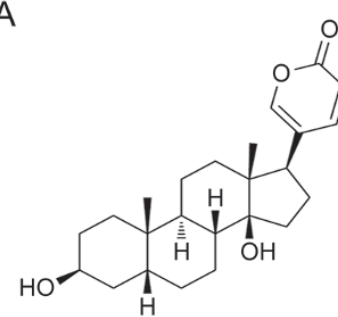

Bufalin (BF)

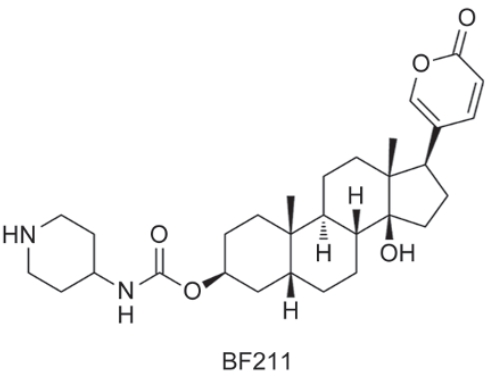

BF211

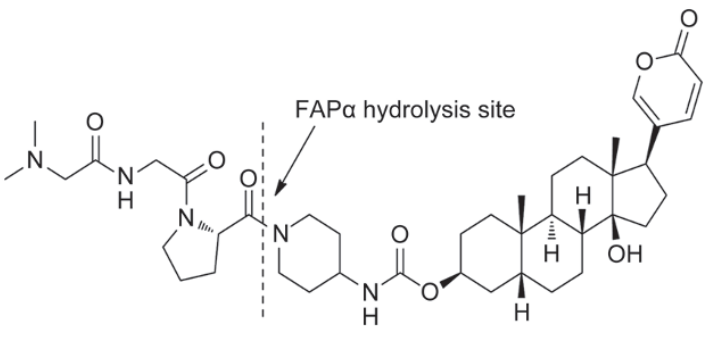

BF211-03

B<smiles>CCOCCN(C)CC(=O)OCc1ccccc1</smiles><smiles>O=C(O)CNC(=O)c1ccccc1</smiles>

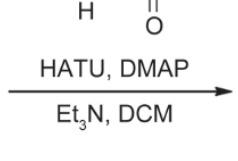

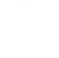$$
\text { (2) }
$$<smiles>CC(C)(C)OC(=O)NCC(=O)N1CCCC1C(=O)OCc1ccccc1</smiles>

Boc-Gly-Pro-Bn<smiles>COC(=O)C(C)OC(=O)CNC(=O)CN(C)C</smiles><smiles>CN(C)CC(=O)NCC(=O)N1CCCC1C(=O)O</smiles>

N,N-dimethyl-Gly-Gly-Pro-Bn

$\mathrm{N}, \mathrm{N}$-dimethyl-Gly-Gly-Pro-OH

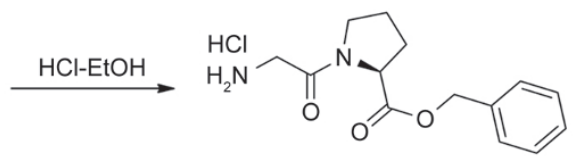

Gly-Pro-Bn
HATU, DMAP,
$\mathrm{Et}_{3} \mathrm{~N}, \mathrm{DCM}$

BF211

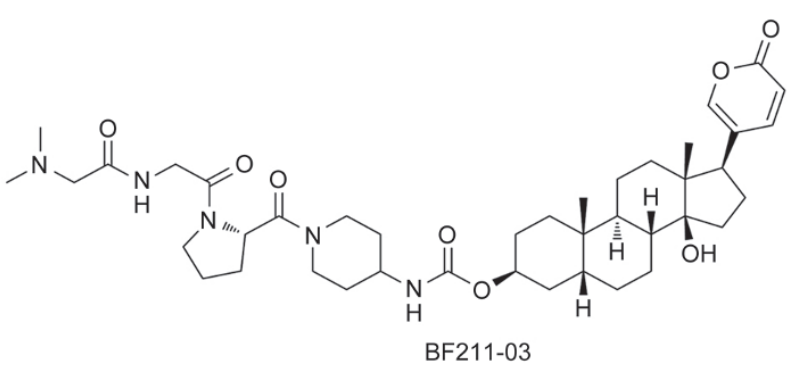

Figure 1. Structures of the compounds and the synthetic route. (A) Structures of BF, BF211 and BF211-03. (B) The synthetic route for preparing the BF211-03 conjugate. 
mice, aged 6-8 weeks, were purchased from the Sino-British SIPPR/BK Lab Animal Ltd (Shanghai, China) and housed in accordance with the protocols approved through the East China Normal University. Generally, on day 0, HCT-116 cells $\left(5 \times 10^{6}\right.$ cells) suspended in $0.1 \mathrm{~mL}$ of PBS were mixed with $0.1 \mathrm{~mL}$ Matrigel basement membrane matrix (BD Biosciences) and subsequently inoculated in the right flank of each mouse. The tumors were grown to $100-150 \mathrm{~mm}^{3}$ before the treatments were administered. The mice were treated with solvent control, BF211 $(7.29 \mu \mathrm{mol} / \mathrm{kg})$ or BF211-03 (14.58 $\mu \mathrm{mol} / \mathrm{kg})$, twice daily via intraperitoneal injection. The tumor size was measured every other day by using calipers, and the tumor volume was calculated with the standard formula width $^{2} \times$ length $/ 2$. Tumor growth inhibition was calculated as $\mathrm{TGI} \%=(1-$ (mean tumor volume of the treatment group on the first day-mean tumor volume of the treatment group on the end day)/(mean tumor volume of the control group on the first day-mean tumor volume of the control group on the end day)) $\times 100 \%$. Body weight was measured every other day.

\section{Statistical analysis}

Student's $t$-test was applied to evaluate the differences between treated and control groups. The data are expressed as the mean \pm SEM, and results from at least 3 independent experiments were used for statistical analysis.

\section{Results}

FAP $\alpha$-dependent release of BF211 from the prodrug BF211-03

As shown in Figure 2, BF211-03 was dose- and time-dependently hydrolyzed to BF211 by rhFAPa. When BF211-03 was incubated with rhFAPa at 2 or $8 \mu \mathrm{g} / \mathrm{mL}$, a decrease in the peak of BF211-03 was accompanied by an increase in the peak of the cleaved product BF211 (Figures 2A and 2B). Furthermore, the BF211 peak was observed after a 1.5-h incubation with $2 \mu \mathrm{g} / \mathrm{mL}$ of $\mathrm{rhFAPa}$, and the peak area increased over time (Figures 2C and 2D). The results suggested that BF211-03 is dose- and time-dependently hydrolyzed by rhFAPa.

Hydrolysis of the prodrug BF211-03 by plasma, tumor tissue homogenates and normal tissue homogenates

To examine the tumor targeting of BF211-03, the hydrolysis of BF211-03 in mouse plasma, tumor tissue homogenate, including HCT-116 tumor xenografts and MGC-803 tumor xenografts, and normal tissue homogenates, including heart and kidney, were analyzed. The protein levels of FAPa in tissue homogenates were assessed. As shown in Figure 3A, high levels of FAPa were observed in tumor homogenates, particularly in HCT-116 tumor tissues, whereas low levels of FAPa were observed in both heart and kidney normal tissue homogenates. In accordance with the levels of FAPa, the cleavage rates of BF211-03 were high in HCT-116 or MGC-803 tumor tissue
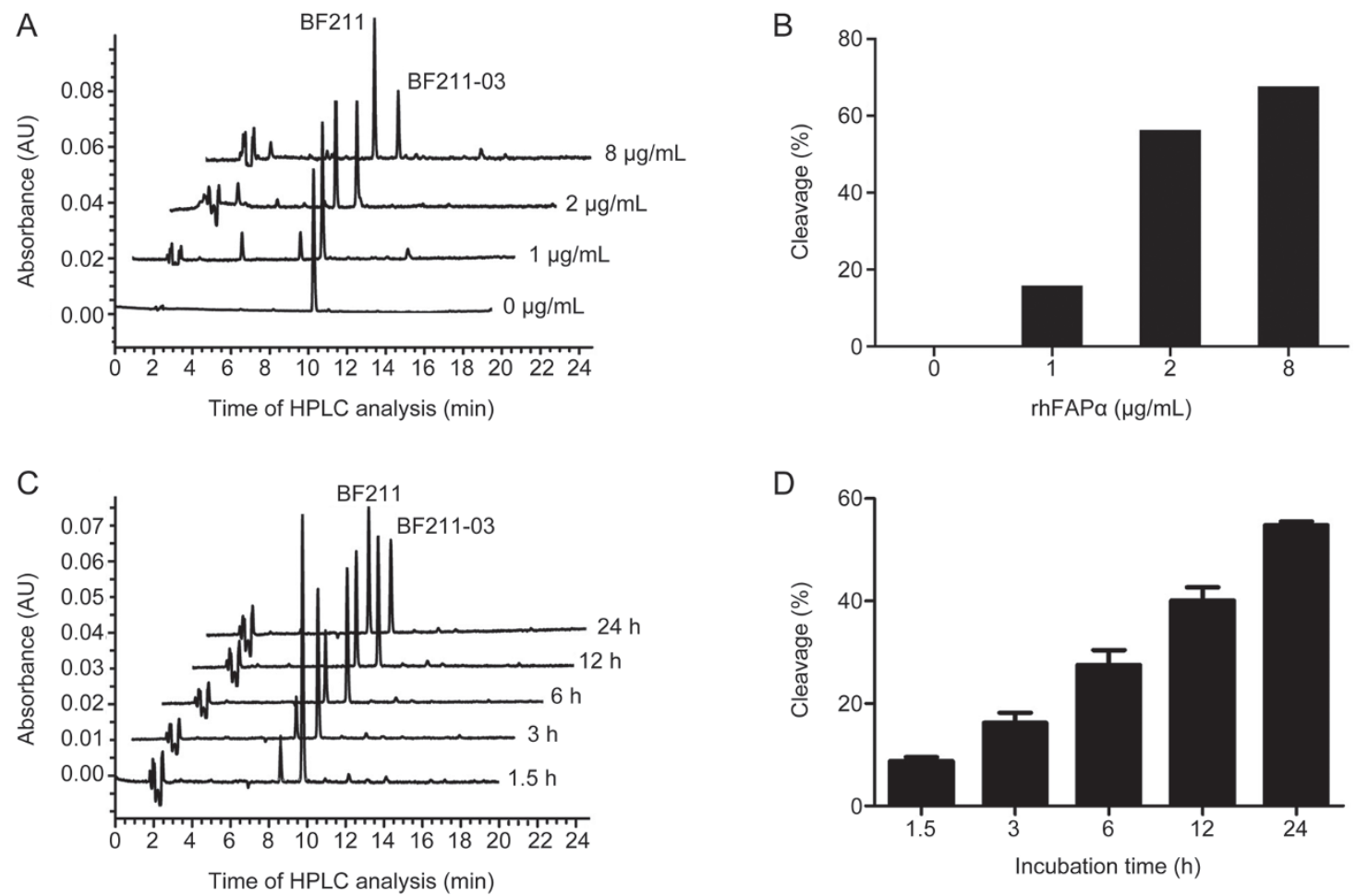

Figure 2. Hydrolysis of BF211-03 by rhFAP $\alpha$. (A) Representative HPLC chromatograms of BF211-03 after incubation with rhFAP $(0,1,2$ and 8 $\mu \mathrm{g} / \mathrm{mL}$ ) for $24 \mathrm{~h}$ at $37^{\circ} \mathrm{C}$. The x-axis shows the time of HPLC analysis, and the $y$-axis shows the peaks for BF211-03 and the cleaved product BF211. (B) Quantitative data for the cleavage of BF211-03 in (A). (C) Representative HPLC chromatograms of BF211-03 after incubation with rhFAP $\alpha$ (2 $\mu g / m L)$ at $37^{\circ} \mathrm{C}$ for $1.5,3,6,12$ or $24 \mathrm{~h}$. (D) Quantitative data for the cleavage of BF211-03 in (C). The data show the statistical results (means \pm SEM, $n=3$ ) of three independent experiments. 
A
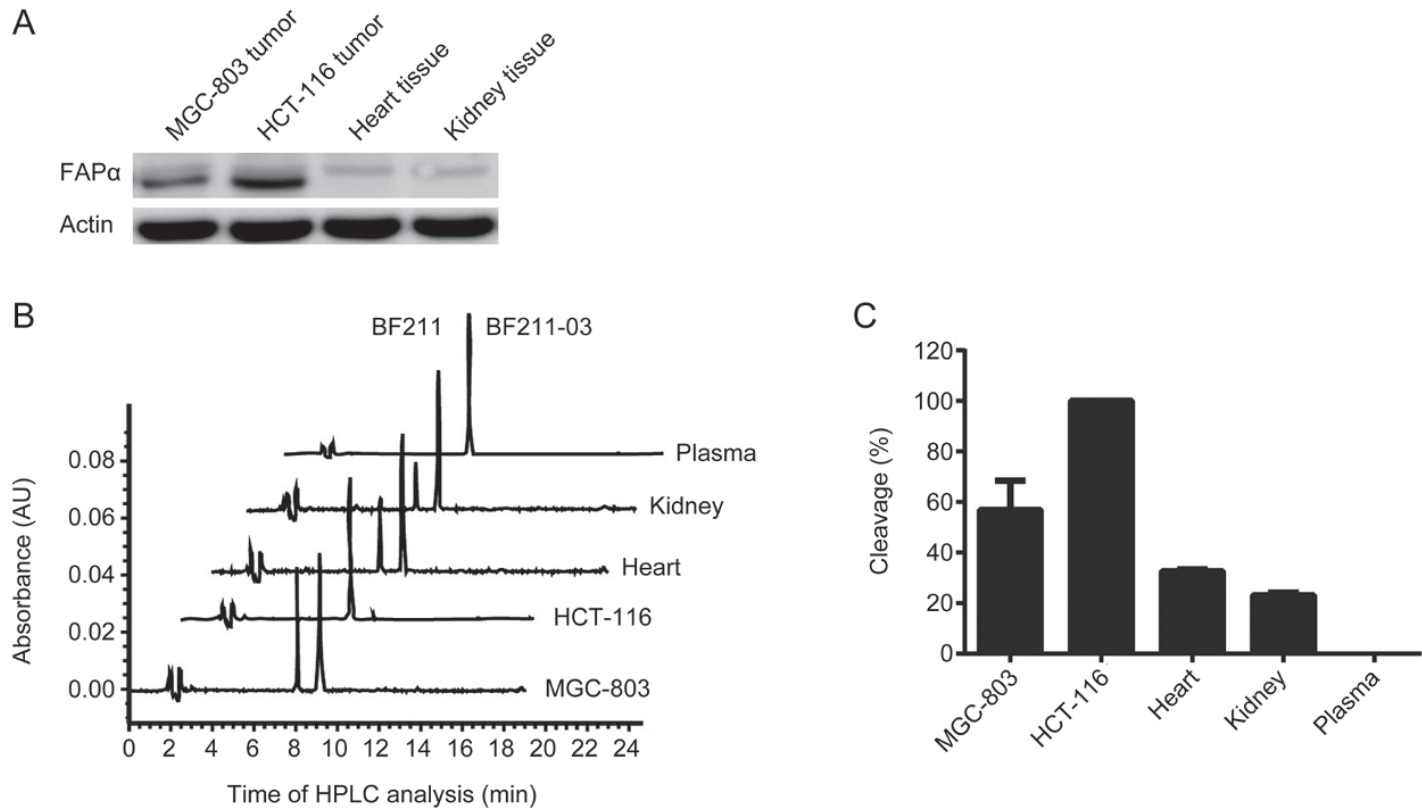

Figure 3. Hydrolysis of BF211-03 by plasma, normal tissue homogenates and tumor tissue homogenates. (A) Western blotting analysis of the expression levels of FAP $\alpha$ in the homogenates of MGC-803 tumor xenografts, HCT-116 tumor xenografts, heart tissue and kidney tissue. Actin was used as a loading control. (B) Representative HPLC chromatograms of BF211-03 after incubation with the homogenates of MGC-803 tumor xenografts, HCT116 tumor xenografts, heart tissue and kidney tissue for $12 \mathrm{~h}$ at $37^{\circ} \mathrm{C}$. The $\mathrm{x}$-axis shows the time of HPLC analysis, and the y-axis shows the peaks for BF211-03 and the cleaved product BF211. (C) Cleavage of BF211-03 after incubation with mouse plasma, normal tissue homogenates and tumor tissue homogenates at $37^{\circ} \mathrm{C}$ for $12 \mathrm{~h}$. The data show the statistical results (means $\pm S E M, n=3$ ) of three independent experiments.

homogenate but markedly lower in heart and kidney tissues (Figures 3B and 3C). No detectable cleavage of BF211-03 was observed after incubation with mouse plasma (Figures $3 \mathrm{~B}$ and 3C). These results suggested that BF211-03 is hydrolyzed to release BF211 in FAPa-positive tumor tissues, but otherwise remains relatively stable in plasma and normal tissues.

\section{Prodrug BF211-03 exhibited lower cytotoxicity than BF211 in} cultured cells

To examine whether the cytotoxicity of BF211 decreased after modification with the Z-Gly-Pro dipeptide, the cytotoxicities of the prodrug BF211-03 and BF211 were compared in cultured HCT-116, MGC-803, MDA-MB-435 and H9C2 cells. First, the expression levels of FAPa in these cells were observed. As shown in Figure 4A, HCT-116 and MGC-803 cancer cell lines had low levels of FAPa expression, whereas, similarly to previously reported findings ${ }^{[20]}$, high levels of FAPa were observed in MDA-MB-435 cells. Thus, MDA-MB- 435 cells were used in the present study to further confirm the capability of hFAPa to hydrolyze BF211-03. Furthermore, the cardiomyocyte line $\mathrm{H} 9 \mathrm{C} 2$ was also used to examine the potential cardiotoxicity of BF211 or BF211-03. The level of FAPa was low in $\mathrm{H} 9 \mathrm{C} 2$ cells (Figure 4A). As shown in Figure 4B, there was a positive correlation between BF211-03 cleavage and the level of FAPa expressed in the cells.

Importantly, as shown in Figures 4C, 4D, 4E and 4F, BF21103 exhibited lower cytotoxicity than BF211 in the cultured cells. Table 1 shows the $\mathrm{IC}_{50}$ values of BF211-03 and BF211 in each cell types. As shown in Table 1, the $\mathrm{IC}_{50}$ value of BF211-
03 in inhibiting HCT-116 cell proliferation was 42.9-fold higher than that of BF211. Similar results were observed with MGC803 cells. For MDA-MB-435 cells with high FAPa expression, only a small difference in the $\mathrm{IC}_{50}$ values of BF211-03 and BF211 was observed (fold difference of only 4.97), a result potentially reflecting the cleavage of prodrug BF211-03 via cellular FAPa. The cytotoxicity of BF211-03 was also lower than that of BF211 in the cardiomyocyte line $\mathrm{H} 9 \mathrm{C} 2$.

Restoration of the cytotoxicity of prodrug BF211-03 after hydrolysis by FAP $\alpha$ or tumor homogenates

To confirm the restoration of cytotoxicity after the hydrolysis of the prodrug, the cytotoxicity of BF211-03 after incu-

Table 1. $\mathrm{IC}_{50}$ values of BF211-03 and BF211 in inhibiting proliferation of cultured cells.

\begin{tabular}{|c|c|c|c|}
\hline \multirow[t]{2}{*}{ Cell line } & \multicolumn{2}{|c|}{$\mathrm{IC}_{50}$ values $(\mathrm{nmol} / \mathrm{L}$, mean $\pm \mathrm{SEM})$} & \multirow[t]{2}{*}{ Fold-difference } \\
\hline & BF211 & BF211-03 & \\
\hline MGC-803 & $0.12 \pm 0.04$ & $5.7 \pm 1.12$ & 48.98 \\
\hline MDA-MB-435 & $3.61 \pm 0.94$ & $17.97 \pm 3.71$ & 4.97 \\
\hline HCT-116 & $0.62 \pm 0.06$ & $26.72 \pm 1.28$ & 42.9 \\
\hline H9C2 & $7800 \pm 1135$ & $30250 \pm 4518$ & 3.88 \\
\hline
\end{tabular}

Inhibitive effects of BF211 and BF211-03 on proliferation of cultured cells were evaluated by CCK8 assay as described in the Materials and Methods. Data were statistical results (mean \pm SEM, $n=3$ ) of three independent experiments. The $\mathrm{IC}_{50}$ fold difference is calculated by dividing the $\mathrm{IC}_{50}$ for cells with BF211-03 by that from BF211-treated cells. 
A

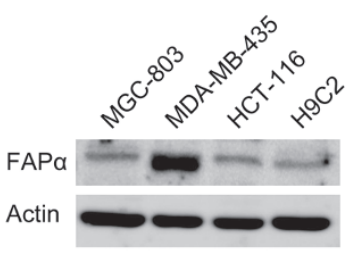

C

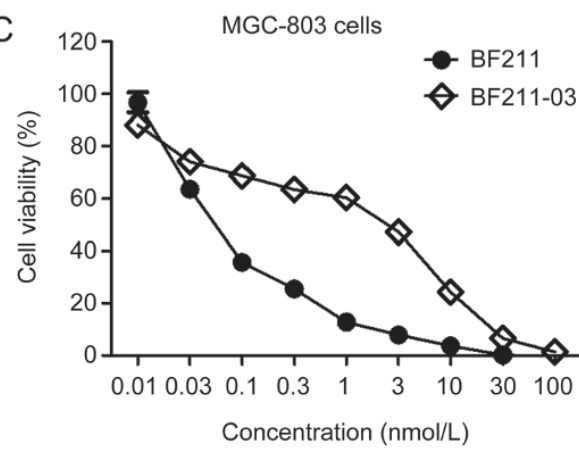

E

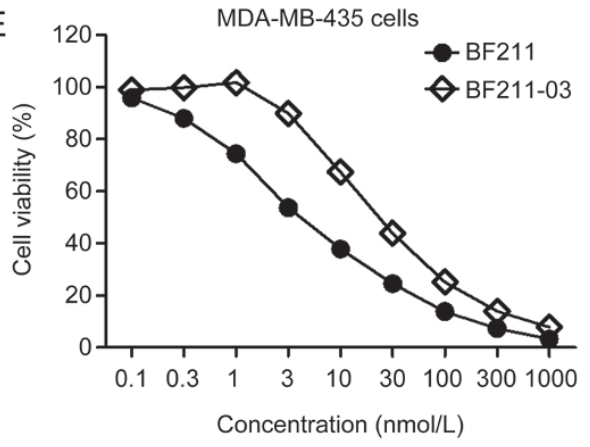

B

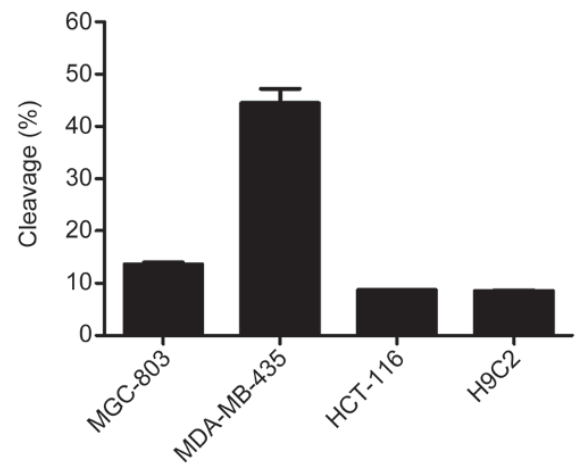

D

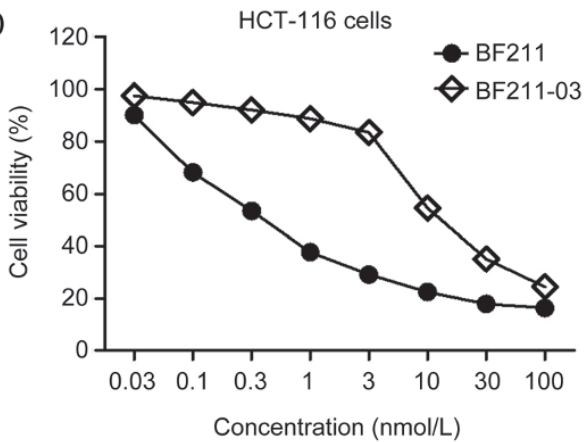

F

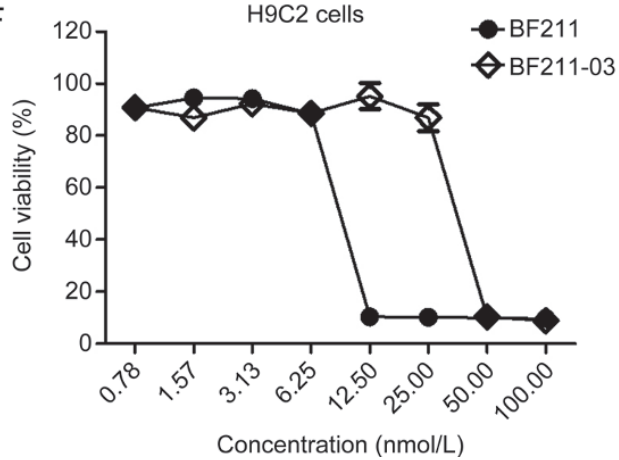

Figure 4. Cytotoxicity of BF211-03 and BF211 in cultured cells. (A) Western blotting analysis of the expression levels of FAP $\alpha$ in MGC-803, MDA-MB-435, HCT-116 and H9C2 cells. Actin was used as a loading control. (B) Cleavage of BF211-03 after incubation with cultured cells at $37^{\circ} \mathrm{C}$ for $8 \mathrm{~h}$. The data show the statistical results (means \pm SEM, $n=3$ ) of three independent experiments. (C-F) Inhibitive effects of BF211-03 and BF211 on proliferation of cultured cells including MGC-803, MDA-MB-435, HCT-116 and H9C2 cells. The data show the statistical results (means \pm SEM, $n=3$ ) of three independent experiments.

bation with rhFAPa or HCT-116 tumor homogenate was assessed. As shown in Figure 5A, rhFAPa-cleaved BF21103 showed higher cytotoxicity than that of un-cleaved BF21103. Notably, the cytotoxicity of BF211-03 cleaved by tumor homogenate was markedly enhanced (Figure 5B). As shown in Table 2, the $\mathrm{IC}_{50}$ value of BF211-03 cleaved by tumor homogenate $(1.89 \pm 0.25 \mathrm{nmol} / \mathrm{L})$ was almost equal to that of BF211 $(1.70 \pm 0.43 \mathrm{nmol} / \mathrm{L})$. These results suggested that the cytotoxicity of prodrug BF211-03 was restored to the level of the parent drug BF211 after incubation with rhFAPa or tumor homogenate.

Prodrug BF211-03 exhibited an antitumor efficacy similar to that of BF211 and elicited a smaller decrease in body weight in nude mice As shown in Figure 6A, BF211-03, at a dose twice that of
BF211, elicited similar inhibitive effects on the tumor growth of HCT-116 xenografts, whereas mice in the BF211-03-treated group displayed a much lower decrease in body weight than that in mice treated with BF211 (Figure 6B). Table 3 shows the data of tumor volumes, TGI and body weight in control, BF211-treated and BF211-03 treated mice. These results suggested that BF211-03 exhibits anti-tumor effects almost equal to those of the parent compound but induces a smaller decrease in body weight.

\section{Discussion}

Traditional Chinese medicines containing ChanSu are still commonly used in clinics for anti-cancer therapy ${ }^{[21,22]}$, and the anti-cancer effects of bufalin have been demonstrated in many reports $^{[23-26]}$. In previous studies ${ }^{[1,2,17,18]}$, derivatives of BF, 

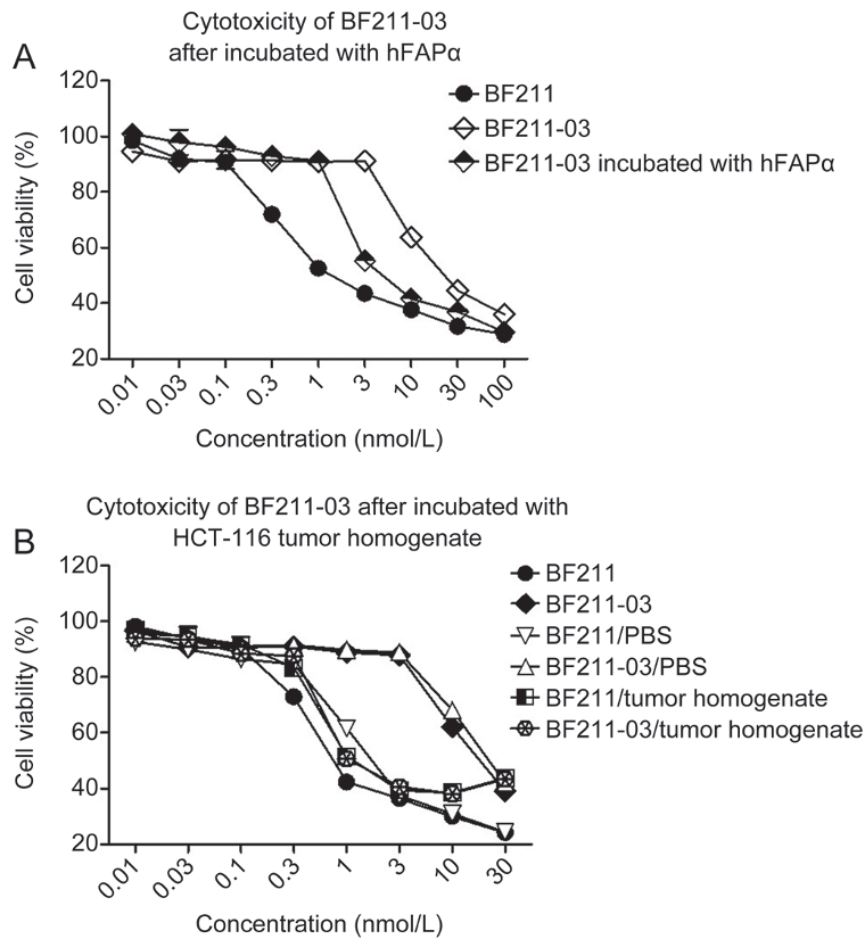

Figure 5. Restoration of cytotoxicity of BF211-03 after hydrolysis by FAP $\alpha$ or tumor homogenate. (A) Cytotoxicity of BF211-03 after incubation with FAP $\alpha(8 \mu \mathrm{g} / \mathrm{mL})$ for $24 \mathrm{~h}$. (B) Cytotoxicity of BF211-03 after incubation with HCT-116 tumor homogenate for $12 \mathrm{~h}$. The data show the statistical results (means \pm SEM, $n=3$ ) of three independent experiments.

such as BF211, have been designed, synthesized and examined for their anti-cancer effects. Compared with BF, BF211 exhibits stronger anti-cancer effects in various cancer cells and lower toxicity in mice ${ }^{[1,2]}$. These results also suggest that BF211 and BF exerts anti-cancer effects by binding with $\mathrm{Na}^{+} / \mathrm{K}^{+}$-ATPase and affecting cellular functions, such as transcription, translation, protein synthesis, proteasome degradation ${ }^{[17,18]}$. Although BF211 exhibits stronger anti-cancer activities and lower systemic toxicity than $\mathrm{BF}^{[1]}$, the development of BF211 as a new anti-can-

Table 2. Cytotoxicity of BF211-03 after hydrolyzed by hFAP $\alpha$ or tumor homogenate.

\section{Chemicals}

BF211

BF211-03

BF211-03/hFAP $\alpha$

BF211/PBS

BF211-03/PBS

BF211/tumor homogenate

BF211-03/tumor homogenate

Cytotoxicity of BF211-03 after hydrolyzed by hFAP $\alpha$ or tumor homogenate was evaluated in HCT-116 by CCK8 assay as described in the Materials and Methods. Data were statistical results (mean \pm SEM, $n=3$ ) of three independent experiments.
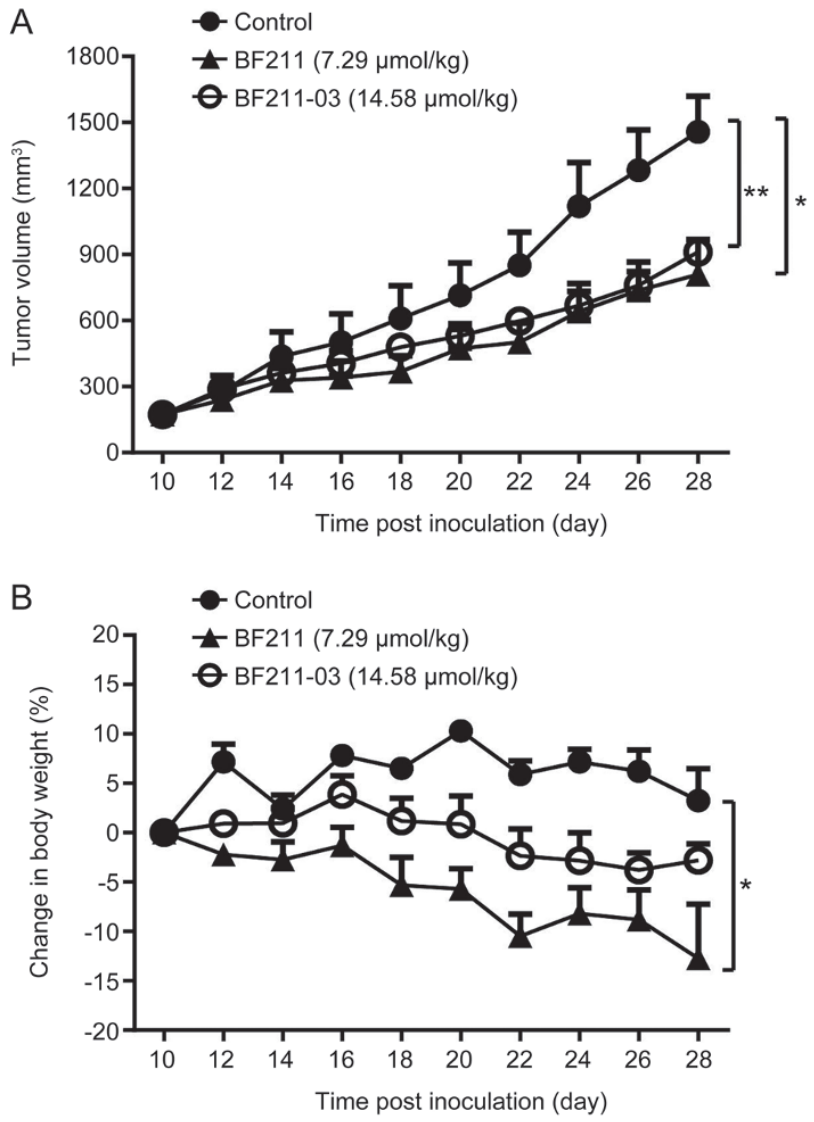

Figure 6. Antitumor efficacy and systemic toxicity of BF211-03 and BF211 in HCT-116 tumor-bearing mice. (A) Tumor volumes in mice during the treatment period. The data show the statistical results (mean \pm SEM, $n=6$ ) of the mice in each group. (B) Changes in body weight in mice during the treatment period. The data show the statistical results (mean \pm SEM, $n=6$ ) of mice in each group. ${ }^{*} P<0.05,{ }^{* *} P<0.01$ vs the control group.

cer agent has been restricted by its potential toxicity in normal tissues, particularly the heart. The cardiotonic effects resulting from $\mathrm{Na}^{+} / \mathrm{K}^{+}$-ATPase inhibition in cardiomyocytes might be one of the major causes of the side effects of BF211 ${ }^{[27-29]}$.

Tumor stroma has gradually emerged as a potential target in anti-cancer therapy ${ }^{[30,31]}$. Compared with traditional approaches for targeting tumor cells, strategies that target tumor stromal cells may offer several potential advantages. First, the targeting of tumor stromal cells is more genetically stable and thus is less likely to acquire resistance to the cytotoxic agent ${ }^{[32]}$. Second, many solid tumor malignancies share common alterations in their tumor microenvironments; therefore, approaches targeting the tumor microenvironment may be widely applied to these different types of tumors ${ }^{[33]}$. FAPa is a membrane-bound protease specifically expressed by reactive stromal fibroblasts, and the potential exploitation of FAPa as a therapeutic target has been assessed. Several studies have reported that FAPa plays an active role in tumor promotion, including increasing in tumor incidence, growth and angiogenesis $^{[14,34,35]}$. Although efforts to directly inhibit the enzymatic activity of FAPa by using antibodies or small molecule inhibitor-based approaches have not been promising ${ }^{[36,37]}$ a 
Table 3. Effects of BF211 and BF211-03 on tumor growth and body weight of mice with HCT-116 xenografts. Data presented are the mean \pm SEM. ${ }^{*} P<0.05,{ }^{* *} P<0.01$ (versus the control group).

\begin{tabular}{|c|c|c|c|c|c|c|c|}
\hline \multirow[t]{2}{*}{ Treatment } & \multicolumn{2}{|c|}{ Tumor volume $\left(\mathrm{mm}^{3}\right)$} & \multirow[t]{2}{*}{ RTV } & \multirow[t]{2}{*}{ TGI (\%) } & \multicolumn{2}{|c|}{ Body weight (g) } & \multirow{2}{*}{$\begin{array}{l}\text { Body weight } \\
\text { change (\%) }\end{array}$} \\
\hline & Start & End & & & Start & End & \\
\hline Control & $174.39 \pm 31.41$ & $1457.40 \pm 162.14$ & $9.33 \pm 1.67$ & - & $19.20 \pm 0.69$ & $19.82 \pm 0.91$ & $3.29 \pm 3.3$ \\
\hline BF211 & $173.96 \pm 32.14$ & $809.87 \pm 159.16^{*}$ & $4.77 \pm 0.49^{*}$ & 50.44 & $20.35 \pm 0.10$ & $17.75 \pm 1.09$ & $-12.72 \pm 5.61^{*}$ \\
\hline BF211-03 & $173.94 \pm 29.13$ & $911.25 \pm 51.58^{* *}$ & $5.93 \pm 1.02$ & 42.53 & $20.38 \pm 0.78$ & $19.78 \pm 0.62$ & $-2.77 \pm 1.70$ \\
\hline
\end{tabular}

RTV: relative tumor volume. TGl: tumor growth inhibition.

FAPa-activated prodrug therapeutic strategy has been used successfully ${ }^{[12-15]}$. The FAPa-activated prodrug strategy relies on the presence and unique post-prolyl endopeptidase activity of FAPa to selectively activate a prodrug in the tumor microenvironment. In the present study, to improve the targeting of BF211, we used a FAPa-activated prodrug strategy involving the design of the prodrug BF211-03. Notably, the peptide used to decorate BF211 was different from the peptides used in previous studies ${ }^{[12-15]}$. In a preliminary study, approximately 20 prodrugs of BF211 were designed and synthesized on the basis of different peptides. By comparing the cleavage rates of these prodrugs under hFAPa treatment, BF211-03, with the highest cleavage rate, was selected for further study. A prodrug used in previous study to modify doxorubicin ${ }^{[15]}$ showed a much lower cleavage rate under hFAPa treatment than that of BF211-03, thus suggesting that different peptides might be used for different parent drugs when prodrugs are designed to be activated by FAPa cleavage, because the structures of both the peptide and parent drug affect the cleavage of the conjugate by FAPa.

The results of the present study showed that BF211-03 can be successfully cleaved to generate BF211 by recombinant FAPa protein or tumor homogenates in vitro, but otherwise remains relatively stable in the plasma and homogenates of normal tissues, including the heart. The similarity between the sequences of human and mice FAPa is approximately $87 \%$, and FAPa is generally absent in most normal human or mouse tissues ${ }^{[38]}$. The results of the present study also showed that the levels of FAPa in normal heart or kidney tissue are low. These results suggested that BF211-03 might be cleaved only in tumor tissues but otherwise remain relatively stable in plasma or normal tissues. Furthermore, the cytotoxicity of BF211 against different types of tumor cells and the cardiomyocyte line $\mathrm{H} 9 \mathrm{C} 2$ was dramatically decreased after chemical decoration in BF211-03, and the cytotoxicity was restored through the hydrolysis of BF211-03 by FAPa protein or tumor homogenates. Therefore, un-cleaved BF211-03 distributed in normal tissues might not exhibit strong toxicity, and BF211 released from the cleavage of BF211-03 by tumor tissues may exert anti-cancer effects. The results of in vivo study also supported the targeted effects of BF211-03. In nude mice with HCT-116 tumor xenografts, BF211-03, at twice the dose used in previous analyses, showed anti-cancer activity similar to that of BF211 but induced a much smaller decrease in the body weight of mice. Compared with BF211, BF211-03 induced a smaller decrease in body weight at its effective dose in anticancer therapy. These findings indicated the improved tumor targeting of BF211 after decoration with the dipeptide Z-GlyPro to generate the prodrug BF211-03. However, the present study was limited to one type of colon xenograft animal model in vivo. The results of the present study suggested only that the FAPa-based prodrug strategy may be used to improve the targeting of BF211. Because the HCT-116 tumor cells used in the present study also expressed FAPa, it is difficult to distinguish whether the enhanced therapeutic index of the BF211 prodrug were resulted from the cancer cells, the carcinomaassociated fibroblasts or both. Additional studies with more xenograft models, such as determining the therapeutic index of BF211-03 in xenografts with no FAPa expression in tumor cells but high FAPa expression in carcinoma-associated fibroblasts, are needed to characterize the properties of BF211-03 and further optimize the prodrug strategy.

Natural products still play important roles in cancer treatment ${ }^{[39-41]}$. Anti-cancer agents from natural products, particularly those with traditional anti-cancer applications, are primarily compounds with cytotoxicity, such as vinblastine, camptothecin, epipodophyllotoxin, and paclitaxel. Therefore, in generating natural products as new anti-cancer agents, it is important to develop prodrug strategies with cancer-targeted delivery and/or release. The results of the present study showed the effectiveness of the FAPa-based prodrug strategy and support additional studies of natural products and their derivatives.

\section{Acknowledgements}

This work was financially supported in part through grants from the Shanghai Science and Technology Innovation Action program (№ 15140904800 and 14431902700), the National Nature Science Foundation of China (№ 81373964 and 81402953), the National Science \& Technology Major Project of China (№ 2014ZX09301-306-03), the Priority Academic Program Development of Jiangsu Higher Education Institutions, and China Postdoctoral Science Foundation (№ 2015T80416) and the Program for Outstanding Scientific and Technological Innovation Team of Jiangsu Higher Education Institutions.

\section{Author contribution}

Xiao-ping CHAI conducted the experiments, analyzed the data 
and drafted the manuscript. Guang-long SUN synthesized BF211-03. Yan-fen FANG participated in the experiments. Li-hong HU and Xuan LIU initiated the research project and revised the manuscript. Xiong-wen ZHANG designed the experiments and revised the manuscript.

\section{References}

1 Liu M, Feng LX, Sun P, Liu W, Wu WY, Jiang BH, et al. A novel bufalin derivative exhibited stronger apoptosis-inducing effect than bufalin in A549 lung cancer cells and lower acute toxicity in mice. PLoS One 2016; 11: e0159789.

2 Lei M, Xiao Z, Ma B, Chen Y, Liu M, Liu J, et al. Synthesis and biological evaluation of bufalin-3-yl nitrogen-containing-carbamate derivatives as anticancer agents. Steroids 2016; 108: 56-60.

3 Ronnov-Jessen L, Petersen OW, Bissell MJ. Cellular changes involved in conversion of normal to malignant breast: importance of the stromal reaction. Physiol Rev 1996; 76: 69-125.

4 Brennen WN, Isaacs JT, Denmeade SR. Rationale behind targeting fibroblast activation protein-expressing carcinoma-associated fibroblasts as a novel chemotherapeutic strategy. Mol Cancer Ther 2012; 11: 257-66.

5 Chen WT, Kelly T. Seprase complexes in cellular invasiveness. Cancer Metastasis Rev 2003; 22: 259-69.

6 Cheng JD, Weiner LM. Tumors and their microenvironments: tilling the soil. Clin Cancer Res 2003; 9: 1590-5.

7 Wu X, Chen X, Zhou Q, Li P, Yu B, Li J, et al. Hepatocyte growth factor activates tumor stromal fibroblasts to promote tumorigenesis in gastric cancer. Cancer Lett 2013; 335: 128-35.

8 Orimo A, Gupta PB, Sgroi DC, Arenzana-Seisdedos F, Delaunay T, Naeem $\mathrm{R}$, et al. Stromal fibroblasts present in invasive human breast carcinomas promote tumor growth and angiogenesis through elevated SDF-1/CXCL12 secretion. Cell 2005; 121: 335-48.

9 Augsten M, Hägglöf C, Olsson E, Stolz C, Tsagozis P, Levchenko T, et al. CXCL14 is an autocrine growth factor for fibroblasts and acts as a multi-modal stimulator of prostate tumor growth. Proc Natl Acad Sci U S A 2009; 106: 3414-9.

10 Servais C, Erez N. From sentinel cells to inflammatory culprits: cancerassociated fibroblasts in tumour-related inflammation. J Pathol 2013; 229: 198-207.

11 De Monte L, Reni M, Tassi E, Clavenna D, Papa I, Recalde H, et al. Intratumor T helper type 2 cell infiltrate correlates with cancerassociated fibroblast thymic stromal lymphopoietin production and reduced survival in pancreatic cancer. J Exp Med 2011; 208: 46978.

12 Akinboye ES, Brennen WN, Rosen DM, Bakare O, Denmeade SR. Iterative design of emetine-based prodrug targeting fibroblast activation protein (FAP) and dipeptidyl peptidase IV DPPIV using a tandem enzymatic activation strategy. Prostate 2016; 76: 703-14.

13 Brennen WN, Rosen DM, Chaux A, Netto GJ, Isaacs JT, Denmeade SR. Pharmacokinetics and toxicology of a fibroblast activation protein (FAP)-activated prodrug in murine xenograft models of human cancer. Prostate 2014; 74: 1308-19.

14 Brennen WN, Rosen DM, Wang H, Isaacs JT, Denmeade SR. Targeting carcinoma-associated fibroblasts within the tumor stroma with a fibroblast activation protein-activated prodrug. J Natl Cancer Inst 2012; 104: 1320-34.

15 Huang S, Fang R, Xu J, Qiu S, Zhang H, Du J, et al. Evaluation of the tumor targeting of a FAPalpha-based doxorubicin prodrug. J Drug Targeting 2011; 19: 487-96.

16 Ma B, Xiao ZY, Chen YJ, Lei M, Meng YH, Guo DA, et al. Synthesis and structure-activity relationships study of cytotoxic bufalin 3-nitrogencontaining-ester derivatives. Steroids 2013; 78: 508-12.

17 Sun P, Feng LX, Zhang DM, Liu M, Liu W, Mi T, et al. Bufalin derivative BF211 inhibits proteasome activity in human lung cancer cells in vitro by inhibiting beta1 subunit expression and disrupting proteasome assembly. Acta Pharmacol Sin 2016; 37: 908-18.

18 Liu M, Feng LX, Sun P, Liu W, Mi T, Lei M, et al. Knockdown of apolipoprotein $\mathrm{E}$ enhanced sensitivity of Hep3B cells to cardiac steroids via regulating $\mathrm{Na}^{+} / \mathrm{K}^{+}-$ATPase signalosome. Mol Cancer Ther 2016; 15: 2955-65.

19 Liu W, Lu Y, Chai X, Liu X, Zhu T, Wu X, et al. Antitumor activity of TY-011 against gastric cancer by inhibiting Aurora A, Aurora B and VEGFR2 kinases. J Exp Clin Cancer Res 2016; 35: 183.

20 Goodman JD, Rozypal TL, Kelly T. Seprase, a membrane-bound protease, alleviates the serum growth requirement of human breast cancer cells. Clin Exp Metastasis 2003; 20: 459-70.

21 Qi F, Li A, Inagaki Y, Kokudo N, Tamura S, Nakata M, et al. Antitumor activity of extracts and compounds from the skin of the toad Bufo bufo gargarizans Cantor. Int Immunopharmacol 2011; 11: 342-9.

22 Meng Z, Yang P, Shen Y, Bei W, Zhang Y, Ge Y, et al. Pilot study of huachansu in patients with hepatocellular carcinoma, nonsmall-cell lung cancer, or pancreatic cancer. Cancer 2009; 115: 5309-18.

23 Lee $\mathrm{CH}$, Shih YL, Lee MH, Au MK, Chen YL, Lu HF, et al. Bufalin induces apoptosis of human osteosarcoma U-2 OS cells through endoplasmic reticulum stress, caspase- and mitochondria-dependent signaling pathways. Molecules 2017; 22.pii: E437.

24 Sousa LQ, Machado KD, Oliveira SF, Araujo LD, Moncao-Filho ED, Melo-Cavalcante AA, et al. Bufadienolides from amphibians: A promising source of anticancer prototypes for radical innovation, apoptosis triggering and $\mathrm{Na}^{+} / \mathrm{K}^{+}$-ATPase inhibition. Toxicon 2017; 127: 63-76.

25 Liu X, Xiao XY, Shou QY, Yan JF, Chen L, Fu HY, et al. Bufalin inhibits pancreatic cancer by inducing cell cycle arrest via the c-Myc/NFkappaB pathway. J Ethnopharmacol 2016; 193: 538-45.

26 Yin PH, Liu X, Qiu YY, Cai JF, Qin JM, Zhu HR, et al. Anti-tumor activity and apoptosis-regulation mechanisms of bufalin in various cancers: new hope for cancer patients. Asian Pac J Cancer Prev 2012; 13: 5339-43.

27 Kwan T, Paiusco AD, Kohl L. Digitalis toxicity caused by toad venom. Chest 1992; 102: 949-50.

28 Cruz Jdos S, Matsuda H. Arenobufagin, a compound in toad venom, blocks $\mathrm{Na}^{+}-\mathrm{K}^{+}$pump current in cardiac myocytes. Eur J Pharmacol 1993; 239: 223-6.

29 Bick RJ, Poindexter BJ, Sweney RR, Dasgupta A. Effects of Chan $\mathrm{Su}$, a traditional Chinese medicine, on the calcium transients of isolated cardiomyocytes: cardiotoxicity due to more than $\mathrm{Na}$, K-ATPase blocking. Life Sci 2002; 72: 699-709.

30 Gjorgieva D, Zaidman N, Bosnakovski D. Mesenchymal stem cells for anti-cancer drug delivery. Recent Pat Anticancer Drug Discov 2013; 8: 310-8.

31 Narunsky L, Oren R, Bochner F, Neeman M. Imaging aspects of the tumor stroma with therapeutic implications. Pharmacol Ther 2014; 141: 192-208.

32 Campbell I, Polyak K, Haviv I. Clonal mutations in the cancerassociated fibroblasts: the case against genetic coevolution. Cancer Res 2009; 69: 6765-8; discussion 69.

33 Pure E. The road to integrative cancer therapies: emergence of a tumor-associated fibroblast protease as a potential therapeutic target in cancer. Expert Opin Ther Targets 2009; 13: 967-73.

34 Poplawski SE, Lai JH, Li Y, Jin Z, Liu Y, Wu W, et al. Identification of selective and potent inhibitors of fibroblast activation protein and 
prolyl oligopeptidase. J Med Chem 2013; 56: 3467-77.

35 Jansen K, Heirbaut L, Cheng JD, Joossens J, Ryabtsova O, Cos P, et al. Selective inhibitors of fibroblast activation protein (FAP) with a (4-quinolinoyl)-glycyl-2-cyanopyrrolidine scaffold. ACS Med Chem Lett 2013; 4: 491-6.

36 Huang Y, Simms AE, Mazur A, Wang S, Leon NR, Jones B, et al. Fibroblast activation protein-alpha promotes tumor growth and invasion of breast cancer cells through non-enzymatic functions. Clin Exp Metastasis 2011; 28: 567-79.

37 Narra K, Mullins SR, Lee HO, Strzemkowski-Brun B, Magalong K, Christiansen VJ, et al. Phase II trial of single agent Val-boroPro (Talabostat) inhibiting fibroblast activation protein in patients with metastatic colorectal cancer. Cancer Biol Ther 2007; 6: 1691-9.

38 Park JE, Lenter MC, Zimmermann RN, Garin-Chesa P, Old LJ, Rettig WJ. Fibroblast activation protein, a dual specificity serine protease expressed in reactive human tumor stromal fibroblasts. J Biol Chem 1999; 274: 36505-12.

39 Aung TN, Qu Z, Kortschak RD, Adelson DL. Understanding the effectiveness of natural compound mixtures in cancer through their molecular mode of action. Int J Mol Sci 2017; 18.pii: E656.

40 Tuorkey MJ. Cancer therapy with phytochemicals: present and future perspectives. Biomed Environ Sci 2015; 28: 808-19.

41 Hon KL, Lee VW. Challenges for drug discovery and development in China. Expert Opin Drug Discovery 2017; 12: 105-13. 